\title{
AN UNUSUAL PRESENTATION OF MALIGNANT MELANOMA
}

\author{
Bari MA, ${ }^{1}$ Thapa $S,{ }^{1}$ Alam $S,{ }^{1}$ Bhuiyan $Z,{ }^{1}$ Mollah $N U,{ }^{1}$ Sharmin $S,{ }^{1} K C A^{2}$
}

${ }^{1}$ Department of Oncology, Banga Bandhu Sheikh Mujib Medical University, Dhaka, Bangladesh, ${ }^{2}$ National Academy

of Medical Sciences, Mahaboudha, Bir Hospital, Kathmandu, Nepal.

\begin{abstract}
Melanoma is one of the aggressive malignancy arising from the melanocyte. Melanoma usually occurs in the mucus membrane and choroid. There are few cases who may present with lymph node, breast and other involvement. However, few may have an atypical origin and rarely the origin may be unknown. Its aggressive presentation, diagnosis and prognosis sometimes is very challenging to the physician. The mainstay of the diagnosis remains the histopathology and immuno-histochemistry (IHC). Varieties of treatment have evolved from surgical excision to systemic chemotherapy and immunotherapy with promising result, the treatment is still challenging. A 30 years old lady presented with lump in her both breasts and generalized lymphadenopathy. Excisional biopsy revealed Diffuse large B cell Non Hodgkin lymphoma (NHL) but IHC was in favour of melanoma. The final diagnosis was cutaneous melanoma of trunk with atypical presentation (generalized lymphadenoapthy and bilateral breast metastasis).
\end{abstract}

\section{KEYWORDS}

Atypical, immunohistochemistry, melanoma, metastasis

\section{CORRESPONDING AUTHOR}

Dr. Sajan Thapa,

Department of Oncology,

Banga Bandhu Sheikh Mujib Medical University,

Email: sajanthp@gmail.com

Orcid No: https://orcid.org/0000-0002-2464-1772

DOI: https://www.doi.org/10.3126/nmcj.v23i1.36238 


\section{INTRODUCTION}

Malignant melanoma is an aggressive tumor arising from melanocyte. Although it comprises only $3.0 \%$ of all skin cancers diagnosed each year, it accounts for approximately $75.0 \%$ of all skin cancer-related deaths. ${ }^{1}$ It usually develops in the mucus membrane and choroid however there are few cases who present with lymph nodal, breast involvement $(<5.0 \%),{ }^{2}$ involvement or metastatic viscera lesions, with no identifiable primary (2.0-3.0 \%), described as melanomas of the unknown primary site (MUPs). ${ }^{3}$ It is common between the age of 15 and 39 years in females and commonly involved sites are the sun-exposed areas including trunk and face. It is also one of the difficult disease to diagnose clinically in early stage and histologically to interpret the complex architecture of the skin biopsies. ${ }^{4}$

Here we are discussing a 30-years old lady who presented to us with atypical presentation of melanoma. The patient had provided written informed consent for the publication.

\section{CASE REPORT}

A 30 years old Asian female, presented with lumps in her both breasts and generalized lymphadenopathy (cervical, axillary, inguinal, occipital). She couldn't remember the precipitating event to the swelling. To begin with, she had noticed a lump on her left breast approximately around 2 months back which was painless, gradually progressive and associated with constant dull aching pain. Subsequently within 2 months, she noticed swelling in her right breast, lower neck, axillary,

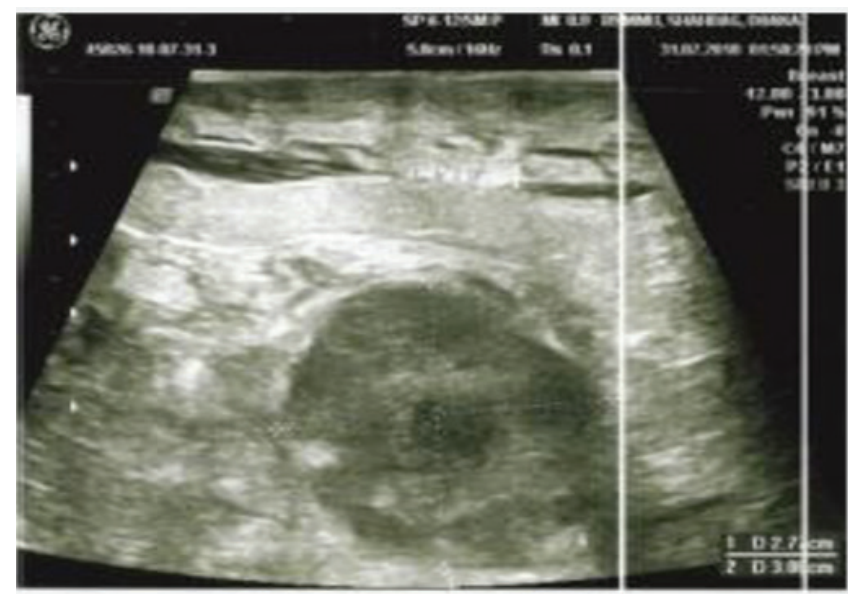

Fig. 1: USG left breast - multiple mixed echogenic area predominantly hypoechoic with irregular outline in the left breast, largest one $3 \mathrm{~cm} \mathrm{X} 2.7 \mathrm{~cm}$.

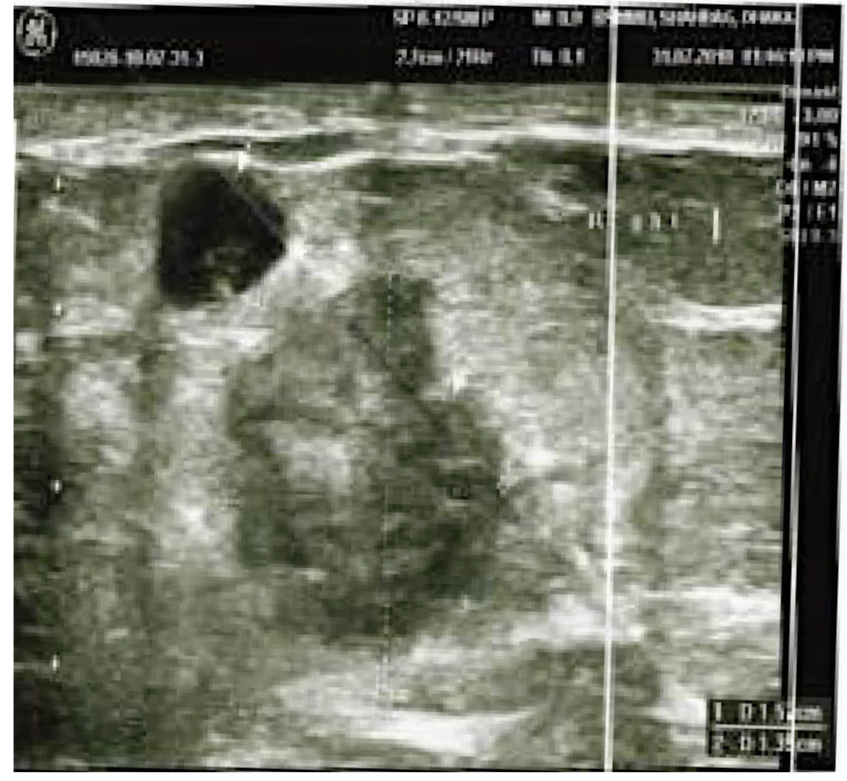

Fig. 2: showing USG of the right breast with multiple mixed echogenic areas predominantly hypoechoic with irregular outline.

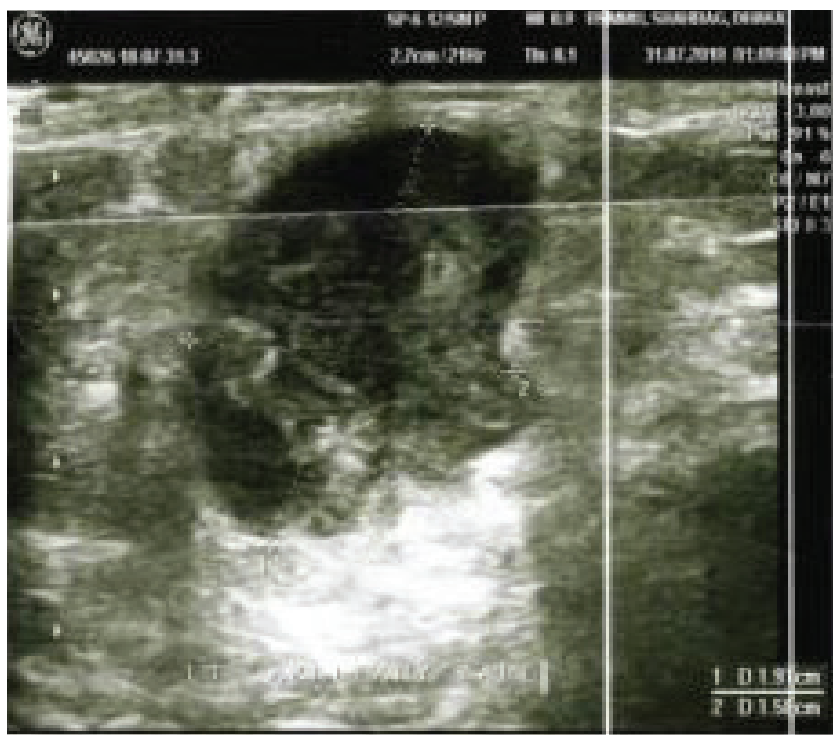

Fig. 3 USG left axillary tail showing a mixed echogenic area with irregular outline measuring about $14.9 \times 1.5 \mathrm{~cm}$ in left axillary tail

inguinal, scalp and upper limbs. There was no history of constitutional symptoms like fever, chills, loss of weight, cough, breathlessness, night sweat, chest pain, or contact with sputum positive tubercular patient. Interestingly on further query, she gave significant past history of excision of a skin lesion (which was painful, gradually progressive and discharging) from the anterior chest wall 3 years back in a rural clinic for which no pathological evaluation was done. 


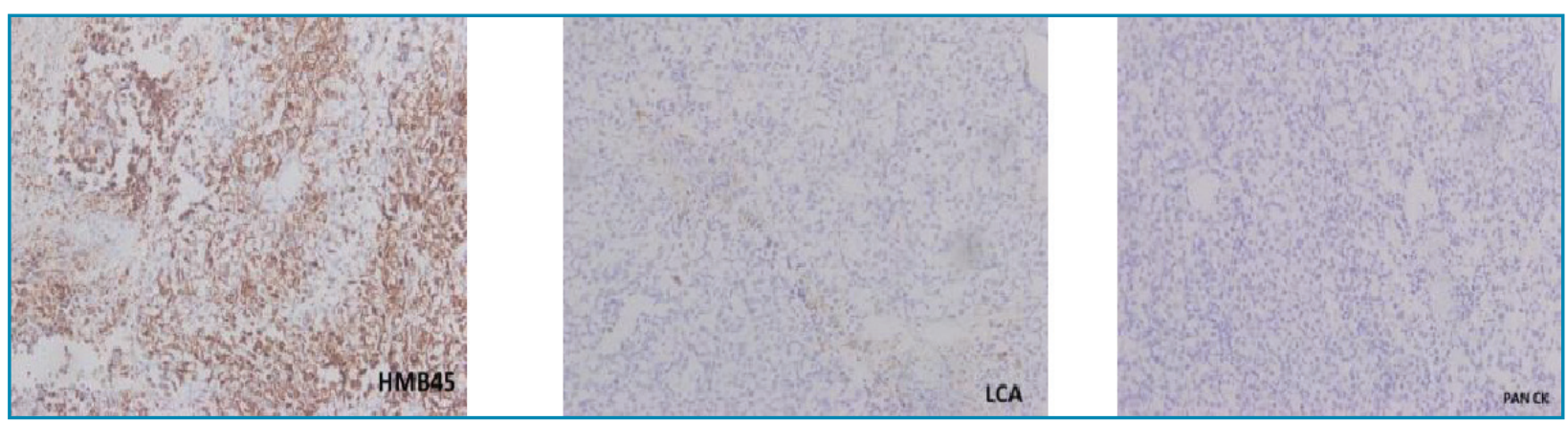

Fig. 4: HMB 45

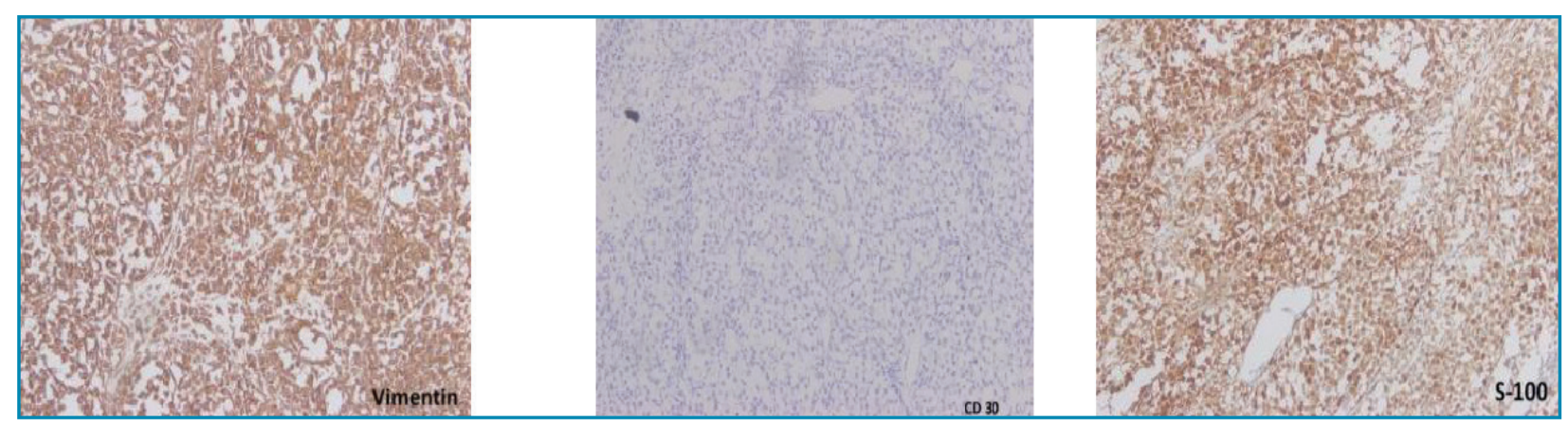

Fig. 5: S100

At presentation she looked ill, (ECOG PS- 3), there were generalized lymphadenopathy (bilateral axillary, left supraclavicular, inguinal region) which were variable in shape and size, largest one in the inguinal region (about $3 \mathrm{~cm}$ $\mathrm{X} 2 \mathrm{~cm}$ in size) firm to hard in consistency, nontender and not associated with discharge. Both breasts were asymmetrical in size and swollen, erythematous with peau'de orange appearance of the skin. There were solitary lumps in both breasts $(9 \mathrm{~cm} \times 7 \mathrm{~cm})$ in the right and $(8 \mathrm{~cm} \times 6$ $\mathrm{cm}$ ) in the left, with a rise in local temperature and tenderness over both breasts. The surface was nodular with irregular margin, firm to hard, fixed with the overlying skin and the underlying structure. Routine blood reports were unremarkable including serology for
HIV, VDRL. Ultrasonography of both breast revealed-multiple SOL's in both breast fig(1, 2 and 3). USG of the abdomen and chest X-ray was unremarkable. FNAC was done from the left breast and left axilla which revealed diffuse NHL. Biopsy was done from the same site which revealed - diffuse high-grade $B$ cell NHL. In the absence of type B symptoms and past history of excision of the skin lesion, Immunohistochemistry (IHC) was done which was suggestive of melanoma (HMB45, Melan A positive) fig ( 4 and 5). With this, a very unusual presentation of melanoma was confirmed. The patient was planned for the immunotherapy however, due to low economical status, cyclophosphamide, vincristine, and dacarbazine (CVD) based regimen was given.
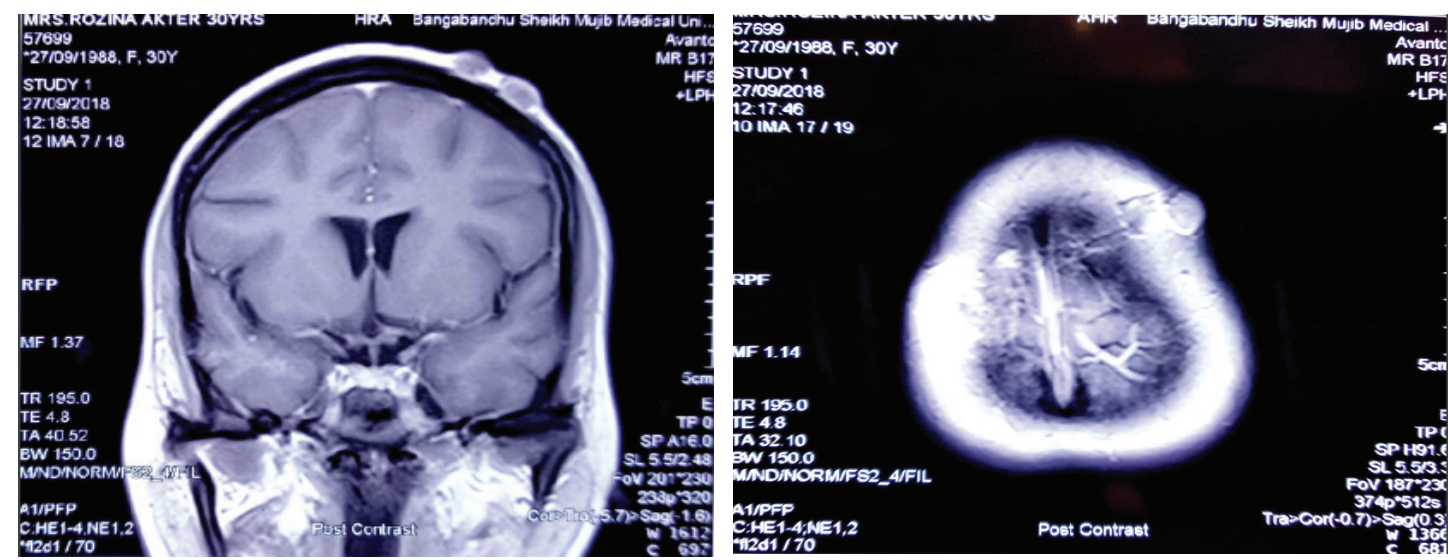

Fig. 6: Brain MRI showing multiple lesions on the scalp over both side of cranium and posterior aspect of neck. 
The patient tolerated 2 cycles of chemotherapy well and there was a slight improvement in the severity of her pain but there were no changes in her functional status, size of the lymph node or breast lump. The condition of the patient did not improve and further deteriorated. After 1-month patient expired.

\section{DISCUSSION}

Our case was a case of a diagnostic dilemma as the patient presented with multiple lymphadenopathies with breast lumps mimicking carcinoma of the breast with lymph node metastasis. But clinical and histopathological evaluation revealed highgrade non-Hodgkin's lymphoma. However IHC and past history led to confirmation of metastatic melanoma.

As per Das Gupta criteria, ${ }^{5}$ our suspicion of malignant melanoma of unknown origin is excluded since we have the evidence of excision of a skin lesion. Hence our case is a case of cutaneous melanoma of the trunk with atypical presentation i.e, generalized lymph nodal and bilateral breast metastasis.

Melanoma arises from the malignant transformation of the melanocyte which produces melanin that is required for pigmentation in the body. Precursor melanocytes develop in the neural crest and, migrate to different areas of the body. ${ }^{6}$ Broadly it can be cutaneous or non-cutaneous. As per the NCCN Database, $91.2 \%$ of melanoma are cutaneous, $5.3 \%$ are ocular, $1.3 \%$ are mucosal and 2-3\% are of unknown primary. ${ }^{7}$ Cutaneous melanomas can occur at any skin site in the body. The most common sites in males are on the back and in the head and neck regions. In women, the most common sites are the lower extremities, commonly below the knee.

Genetic and environment are suspected to be causative factors. Genetic factors include the inheritance of sun-sensitive skin (fair skin type and susceptibility to sunburn) and specific melanoma-related genes (CDKN2A, which encodes the P16 and p14ARF proteins). ${ }^{7}$

Both lymphatic, as well as less likely hematogenous metastasis, has been identified in melanoma and these are notorious to metastasize virtually in any distant site. ${ }^{3}$ The management of melanoma is surgical if the disease is localized and surgically feasible. However, in our case of truncal melanoma, due to the interrelated connections of the lymphatics of the trunk, it is difficult to determine which lymph node basin is the primary site of drainage. Treatment includes removal of primary site lesion, intervening lymphatics, and primary echelon of lymph nodes to which metastases may occur.

She was presumed to be the case of lymphoma or carcinoma of the breast with multiple lymph node metastases. Despite histopathological features of Non-Hodgkin's lymphoma which was contradictory to the clinical presentation, the IHC report was done for the confirmation of melanoma. Though, the primary site of origin remained unidentified, the history of excision of the discharging skin lesion well supported the dagnosis of melanoma. This highlights the possibility of variable, paradoxical, challenging presentation of the melanoma and the value of thorough extensive history and the optimally curtailed investigations to yield the diagnosis.

\section{REFERENCES}

1. Sharma K, Mohanti BK, Rath GK. Malignant melanoma: A retrospective series from a regional cancer center in India. J Cancer Res Ther 2009; 5: 173-80.

2. Kurul S, Taş F, Büyükbabani N, Mudun A, Baykal C, Çamlıca H. Different Manifestations of Malignant Melanoma in the Breast: a Report of 12 Cases and a review of the literature. Japanese J Clinl Oncol 2005; 35: 202-6.

3. Kamposioras K, Pentheroudakis G, Pectasides D, Pavlidis N. Malignant melanoma of unknown primary site. To make the long story short. A systematic review of the literature. Crit Rev Oncol/Hematol 2011; 78: 112-6.

4. Magro CM, Crowson AN, Mihm MC. Unusual variants of malignant melanoma. Mod

Pathol 2006; 19 (Suppl 2): S41-S70. doi: 10.1038/ modpathol.3800516.

5. Dasgupta T, Bowden L, Berg JW. Malignant melanoma of unknown primary origin. Surg Gynecol Obstet 1963; 117: 341-5.

6. DeVita V, Lawrence T, Rosenberg S. DeVita, Hellman, and Rosenberg's cancer. Philadelphia: Lippincott Williams \& Wilkins; 2015.

7. Anbari K, Schuchter L, Bucky L et al. Melanoma of unknown primary site. Cancer 1997; 79: 181621.

8. Hussussian C, Struewing J, Goldstein A et al. Germline p16 mutations in familial melanoma. Nature Genetics 1994; 8: 15-21. 